\title{
Gender und der überfrachtete kolumbianische Frieden
}

\author{
Anika Oettler
}

Eingegangen: 5. Juni 2020 / Überarbeitet: 18. August 2020 / Angenommen: 15. Dezember 2020 / Online publiziert: 7. Januar 2021

(C) Der/die Autor(en) 2021

Zusammenfassung Das 2016 unterzeichnete Friedensabkommen zwischen der kolumbianischen Regierung und der FARC-EP hat sowohl in prozeduraler als auch in substantieller Hinsicht neue Maßstäbe für inklusive Friedensprozesse gesetzt. Der Beitrag konzentriert sich auf die kontroverse Einbeziehung einer Vielfalt von genderbezogenen Maßnahmen in den Vertragstext, die das Ergebnis von transnationalen Normentwicklungen und lokalen Kämpfen ist. Im Mittelpunkt des Beitrages steht der Vertragstext selbst. Es wird zunächst aufgezeigt, wie sich die Debatte um eine angeblich vorhandene Gender-Ideologie nach dem Scheitern der Volksabstimmung auf die revidierte Fassung des Abkommens ausgewirkt hat: der Begriff wurde vermieden und ein traditionelles Geschlechterverständnis gestärkt, ohne dabei jedoch die Vielzahl von Einzelmaßnahmen zu reduzieren. Die hier vorgeschlagene qualitative Systematik der genderbezogenen Einzelmaßnahmen zeigt auf, dass es sich überwiegend um Maßnahmen zur Bearbeitung von Konfliktursachen mit einem geringen Konkretisierungsgrad handelt. Dies geht mit einer bisher geringen Implementierung in diesen Feldern einher. Der Beitrag zeigt, wie mit einer prioritätslosen Fülle von genderbezogenen Einzelmaßnahmen leicht ein Umschlagpunkt erreicht wird, an dem sich das eigentliche Anliegen feministischer Bewegungen - die strukturellen Konfliktursachen anzugehen - verliert.

Schlüsselwörter Kolumbien · Friedensabkommen · Friedensprozess · Inklusivität · Gender

\footnotetext{
A. Oettler $(\bowtie)$

Philipps-Universität Marburg, Ketzerbach 11, 35032 Marburg, Deutschland

E-Mail: oettler@staff.uni-marburg.de
} 


\title{
Gender and Colombia's fraught peace
}

\begin{abstract}
The 2016 peace agreement between the Colombian government and the FARC-EP marks an important procedural and substantial milestone on the road to inclusive peace. The paper focuses on Colombia's highly controversial gendered agenda for peace, a product of transnational norms and local struggles. The preplebiscite and post-plebiscite versions of the peace accord are examined as a site where the interplay of competing interests becomes manifest. The paper shows that the Colombian debate on "gender ideology" and the failed referendum led to readjustments within traditional gender norms, without reducing the broad range of gender-related measures. The systematic qualitative assessment of gender-related measures presented here demonstrates that there is a predominance of rather vague measures related to the roots of violent conflict. Accordingly, there is a slow pace of implementation in these fields. The paper argues that a broad peace agenda including an extensive non-prioritized portfolio of gender-related measures could lead to a tipping point when the core demand of feminist movements to address the root causes of violent conflict fades.
\end{abstract}

Keywords Colombia $\cdot$ Peace accords $\cdot$ Peace process $\cdot$ Inclusive peace $\cdot$ Gender

\section{Einleitung}

In der kolumbianischen Geschichte der Friedenssuche liegen zwei Erfahrungen eng beieinander: die Hoffnung auf eine bessere Zeit und die Enttäuschung über die Persistenz der Gewalt. Vor vier Jahren schimmerte durch das Friedensabkommen zwischen der kolumbianischen Regierung und der Guerilla FARC-EP (Fuerzas Armadas Revolucionarias de Colombia - Ejército del Pueblo, dt.: Bewaffnete Revolutionäre Streitkräfte Kolumbiens - Armee des Volkes) die Morgenröte eines friedlichen Wandels. Der vorangegangene Verhandlungsprozess stellte sowohl in prozeduraler als auch in substantieller Hinsicht einen der bis dato ambitioniertesten Versuche dar, die Konturen eines transformativen Friedens zu zeichnen. Dies war indes keineswegs unumstritten und die Gesellschaft zeigte sich in dieser Frage zerrissen und polarisiert. Am 02.10.2016 stimmte eine hauchdünne Mehrheit von 50,21\% der zur Volksabstimmung angetretenen Kolumbianer*innen gegen das Friedensabkommen.

Die Debatte um eine angeblich vorhandene „Gender-Ideologie“ war zwar nicht für den Wahlausgang ausschlaggebend, spielte aber doch eine wichtige Rolle in der öffentlichen Auseinandersetzung mit dem Friedensprozess (Oettler 2016; Beltrán und Creely 2018). Dabei hatten Befürworter*innen und Gegner*innen des Friedensabkommens gleichermaßen erkannt, dass sich die Frage der Geschlechterverhältnisse - wie auch die Inklusion vulnerabler sozialer Gruppen und die Überwindung regionaler Disparitäten - als Querschnittsthema durch den gesamten Vertragstext zog. Die Verankerung einer Gender-Perspektive war sowohl Ausdruck von transnationalen Normbildungsprozessen im Bereich von Peacebuilding als auch das Ergebnis 
jahrzehntelanger Kämpfe von Frauen- und LGBT-Organisationen ${ }^{1}$, feministischen Gruppierungen und lokalen NGOs. Es waren auch diese Kräfte, die dafür sorgten, dass die Gender-Perspektive - wenn auch in modifizierter Form - in der überarbeiteten Fassung des Friedensabkommens als ein integraler Bestandteil beibehalten wurde. Diese überarbeitete Fassung wurde der Bevölkerung nicht erneut zur Abstimmung vorgelegt, sondern im November 2016 in Bogotá unterzeichnet.

Im Mittelpunkt des vorliegenden Beitrages steht der Text des Friedensabkommens, der als das Ergebnis des Ringens um eine gültige Wirklichkeit analysiert wird. Während zu der prozeduralen Dimension der Gender-Debatte im jüngsten kolumbianischen Friedensprozess bereits einige Arbeiten vorliegen (Esguerra Muelle 2017; Céspedes-Baéz und Jaramillo Ruiz 2018; Oettler 2019), steht eine detaillierte kritische Lektüre der prä- und post-plebiszitären Versionen des Friedensabkommens sowie eine qualitative Systematik der genderbezogenen Maßnahmen des Abkommens noch aus. Dieser Beitrag will beides liefern, indem der Genderdiskurs des Friedensabkommens als ein Bündel von „Aussagen [untersucht wird], die sich hinsichtlich eines bestimmten Themas systematisch organisieren und durch eine gleichförmige (nicht identische) Wiederholung auszeichnen“ (Landwehr 2008, S. 92-92). Methodisch orientiert sich der vorliegende Beitrag am Vorgehen der kritischen Diskursanalyse (Jäger 2004). Der Vertragstext wird als ein Dokument analysiert, in das sich seine von ideologischen Kämpfen, Verhandlungen und nicht-diskursiven Auseinandersetzungen geprägte Entstehungsgeschichte unmittelbar einschreibt. Aus der Perspektive der kritischen Diskursanalyse ist ferner der Umstand relevant, dass das Friedensabkommen einen Katalog des Sagbaren und Gültigen bietet, der mit der Intention ausgehandelt wurde, zukünftige diskursive und nicht-diskursive Praxen zu strukturieren, zu ermöglichen und einzugrenzen. Der Vertragstext wurde einer Strukturanalyse unterzogen, im Hinblick auf den Genderdiskurs an ausgewählten Stellen feinanalytisch in den Blick genommen und schließlich - im Hinblick auf die konkreten genderbezogenen Maßnahmen - auf der Grundlage vorliegender Systematiken qualitativ strukturiert. $^{2}$

Ausgewertet und verglichen wurden erstens die beiden Versionen des Friedensvertrages, um nachzuvollziehen, wie die Debatte um die „Genderideologie“ in den sehr kurzen Überarbeitungsprozess des Friedensabkommens Eingang gefunden hat. Deutlich wird, dass ein binäres Geschlechterverständnis gestärkt wurde (CéspedesBaéz 2017), andererseits aber keine Streichungen im Portfolio der genderbezogenen Maßnahmen vorgenommen wurden. Das im November 2016 in Bogotá unterzeichnete Friedensabkommen enthält das gleiche unüberschaubare Geflecht von Einzelmaßnahmen wie die wenige Wochen zuvor in Cartagena gefeierte Version.

Was bei einer Analyse der Genderdimension des Abkommens allerdings beachtet werden sollte, ist der Umstand, dass das Abkommen insgesamt überkomplex

\footnotetext{
1 Das Akronym LGBT (lesbian, gay, bisexual, transgender) wird in diesem Aufsatz als diejenige Abkürzung verwendet, die in Kolumbien im Kontext staatlicher Politik und zivilgesellschaftlicher Advocacy gebräuchlich ist. S. ausführlich Abschnitt 2.

2 Außerdem wurden bei regelmäßigen Forschungsaufenthalten in Kolumbien zahlreiche Hintergrundgespräche mit Aktivist*innen, Wissenschaftler*innen und Teilnehmer*innen von Friedensverhandlungen geführt.
} 
ist. Das mit der Beobachtung und Dokumentation der Implementierung des Friedensabkommens beauftragte Kroc Institute (2019) hat insgesamt 578 Maßnahmen identifiziert, von denen 130 einen Gender-Bezug aufweisen. In diesem Zusammenhang ist zweierlei zu unterstreichen. Erstens lassen sich die hier vorlegten Überlegungen ebenso auf das Gros der nicht-genderbezogenen Bestimmungen des Friedensabkommens anwenden. Zweitens beruht die hier entwickelte Argumentation auf einem queer-feministischen Grundverständnis, das Identitätslogiken, Machtverhältnisse und Inklusions-/Exklusionsprozesse hinterfragt. Diesem Grundverständnis zu folgen, ist ein Balanceakt, denn es geht darum, zu zeigen, dass mit einer prioritätslosen Fülle von gender-bezogenen Einzelmaßnahmen leicht ein Umschlagpunkt erreicht wird, an dem sich das eigentliche Anliegen feministischer Bewegungen - die strukturellen Konfliktursachen anzugehen - verliert.

Im Folgenden werden zunächst begriffliche Grundlagen (2.) und der internationale Kontext der feministischen Friedenspolitik skizziert (3.). Daran schließen sich eine kurze Geschichte des Friedensprozesses (4.) und der Friedensverhandlungen in Havanna (5.) an. Sodann werden die beiden - prä- und postplebiszitären - Versionen des Friedensvertrages in Bezug auf die Gender-Perspektive durchleuchtet (6.), die gender-bezogenen Bestimmungen des Vertragstextes systematisiert (7) und die Frage der Implementierung diskutiert (8.). Im Fazit der Arbeit werden die Problematiken der Akzeptanz einer feministischen Friedensagenda und der Überfrachtung inklusiver Friedensabkommen nochmals aufgegriffen und eingeordnet.

\section{Terminologische Klärungen}

Die kolumbianischen Debatten über Friedensabkommen und gender-sensible Formen der Konflikttransformationen bewegen sich in einem konzeptionellen Feld, das sich zwischen zwei entgegengesetzten theoretischen Positionen aufspannt. Die eine steht in der Tradition des biologischen Determinismus und betont das natürlich Gegebene zweier Geschlechter. In dieser Lesart gibt es Männer und Frauen mit je spezifischen Bedürfnissen und Interessen. Gender findet nicht als kritischer Gegenentwurf Verwendung, der das gesellschaftlich Konstruierte betont, sondern als Synonym für biologisch geprägtes Geschlecht. LGBT taucht als Sammelkategorie für ein Drittes auf, das doch immer wieder auf die binäre Grundordnung zurückführt. Die andere Tradition ist die der kritischen Gender Studies, die ein dialektisches Verhältnis von sex und gender benennt und gender relational begreift (Connell 1987). Unter Bezugnahme auf dekonstruktivistische und post-essentialistische Ansätze in Philosophie und Psychologie werden Marginalisierungen und Machtverhältnisse offengelegt und das Prozesshafte von Identitätsbildungen betont. Im Fokus dieser kritischen Perspektive stehen Gewaltverhältnisse und normative und kulturelle Setzungen im Rahmen patriarchaler Ordnungen. Im Anschluss an Connell wird das Zwei-GeschlechterModell aufgebrochen und durch den Blick auf diverse Maskulinitäten und Weiblichkeiten ersetzt. In dieser Lesart ist das Akronym LGBT unzureichend, da es das breite Spektrum von nicht-heteronormativen Lebensweisen und Identitäten sowie die unterschiedliche Betroffenheit von patriarchalen Verhältnissen nicht abzubilden vermag. Vor diesem Hintergrund finden im deutschsprachigen Raum Abkürzungen 
wie LGBTIQA+ (lesbian, gay, bisexual, transgender, intersex, queer/questioning, asexual und andere nicht-binäre Identitäten) und FLINT* (Frauen*, Lesben, inter, non-binary und trans* Personen) Verwendung. In Kolumbien ist LGBT das auch lautsprachlich am meisten verwendete Akronym. Während LGBTQ+ zudem in akademischen Zirkeln und studentischen Zusammenhängen verwendet wird, verdankt sich der Gebrauch von LGBTI einer Gerichtsentscheidung zur LGBTI-Politik (Castillo Vargas 2019, S. 21). Wenn im Folgenden die Abkürzung LGBT verwendet wird, geht es folglich darum, der Begriffswahl von kolumbianischen Politiker*innen und NGOs wie Colombia Diversa und Caribe Afirmativo Rechnung zu tragen. Es handelt sich also um ein strategisch verwendetes Akronym, hinter dem das Bewusstsein um Pluralität steht.

Für das im Folgenden entwickelte Argument ist die begriffliche Klärung relevant, weil die Debatte um eine gender-sensible Friedensagenda auch eine ist, in der verschiedene Begrifflichkeiten in Stellung gebracht werden. Die zwei konzeptionellen Pole - ein biologistisch-deterministisches Zwei-Geschlechter-Modell und ein de-essentialistisches Verständnis von gender als facettenreicher sozialer Konstruktion, die im Rahmen patriarchaler Verhältnisse immer neu ausgehandelt wird - kommen dabei in unterschiedlichen, oft auch paradoxen Diskursallianzen zum Zuge. Entscheidend ist dabei der Umstand, dass sowohl in den zivilgesellschaftlichen Diskursräumen der Frauen-, LGBT- und feministischen Bewegungen als auch im Aktionsfeld staatlicher Politik verschiedene, konservativ-autoritäre, paritätsorientierte, liberale, diskriminierungssensible, intersektionale und radikale Konzeptionen eine Rolle spielen. Die Debatte über die Dekonstruktion hegemonialer militärischer Männlichkeiten (Theidon 2009) hat zum Beispiel bereits in den 2010er Jahren Eingang in den offiziellen Diskurs über DDR (Disarmament, Demobilzation, Reintegration) gefunden. Auf der anderen Seite zeigt die Geschichte des jüngsten Friedensabkommens, wie eine breite und intersektional informierte Genderagenda auf ein additives Verständnis von Betroffenheit reduziert werden kann. Zugleich schwingt aber - wie im Folgenden zu zeigen sein wird - auch hier das Potenzial einer emanzipatorischen Friedenspolitik mit.

\section{Resolution 1325 und feministische Friedenspolitik}

Für ein Verständnis der kolumbianischen Debatten um ein inklusives und gendersensibles Friedensabkommen ist zunächst der Blick auf globale Entwicklungen entscheidend. Dass „Gender“ für die Konfliktregulierung durch Friedensabkommen und die Erarbeitung von Maßnahmenkatalogen zur langfristigen Friedenskonsolidierung nicht nur relevant, sondern auch mainstreamfähig wurde, ist eine Entwicklung der 1990er-Jahre. ${ }^{3}$ Obwohl die Geschichte dieser Debatten und der damit zusammenhängenden politischen Kämpfe viel weiter in die Vergangenheit zurückreicht, erreichte

\footnotetext{
3 Zur historischen Einordnung ist die Debatte um die Bedeutung von Geschlechterverhältnissen in der deutschsprachigen Friedensforschung aufschlussreich, die ebenfalls erst in den späten 1980er-Jahren sichtbar wurde; so charakterisiert Ulrike Wasmuth (1998) einen Beitrag von Tordis Batschneider auf dem „Perspektivenkolloquium“ der Arbeitsgemeinschaft Friedens- und Konfliktforschung (AFK) 1989 als wichtiges Diskursereignis im diesem Zusammenhang. Obwohl weder die deutsche Friedensforschung der wichtigste
} 
Frauen- und Genderpolitik erst nach dem Ende der Systemkonfrontation auf der internationalen Ebene „eine gewisse institutionelle Relevanz“ (Ruppert 1998, S. 15). Dies ging einher mit einem veränderten Sicherheitsbegriff, der in den 1990er-Jahren in Gestalt der „menschlichen Sicherheit“ ebenfalls in die Sphäre der institutionellen internationalen Politik einzog. Auch im Feld der Friedenspolitik erhielt die Einsicht, dass die Beendigung von bewaffneten Konflikten nur den ersten Schritt auf dem Weg zu einem dauerhaften und stabilen Frieden markiert, zunehmende Bedeutung. In diesem Kontext waren die Entwicklungen in Zentralamerika, wo das Gipfeltreffen „Esquipulas II“ im Jahr 1987 den Weg verhandelter Konfliktbeilegungen vorzeichnete, wegweisend. 1990 fanden in Guatemala entscheidende Gespräche zwischen der Regierung und dem Guerillaverband URNG (Unidad Revolucionaria Nacional de Guatemala, dt: Revolutionäre Nationale Einheit Guatemalas) statt, in denen sowohl die Modi als auch die Konturen der Friedensgespräche diskutiert wurden. Dabei wurden sowohl die Vorstellung von substanziellen Verhandlungen, die das soziale und politische Gerüst der guatemaltekischen Gesellschaft adressierten, als auch die Forderungen nach öffentlichen Verhandlungen und der Einbeziehung der Zivilgesellschaft artikuliert (Oettler 2004, S. 76-77). Ihren institutionellen Ausdruck fand letztere in der Einsetzung der ASC (Asamblea de la Sociedad Civil, dt. Versammlung der Zivilgesellschaft), die aus Vertreter*innen von zehn zivilgesellschaftlichen Sektoren bestand. Für die guatemaltekischen Verhältnisse der 1990erJahre war der Umstand bemerkenswert, dass auch „Frauen“ als einer der Sektoren eine Stimme hatten. Die Diskussionen innerhalb der ASC trugen maßgeblich dazu bei, dass die Interessen und Betroffenheit von Frauen - wenn auch nicht sehr prominent - Eingang in die Vertragstexte fanden. Der guatemaltekische Fall verweist auf eine doppelte Entwicklung. Auf der prozeduralen Ebene gewannen in den 1990erJahren Schlagworte wie „Mehrebenenpolitik“, local ownership, „Partizipation“ und empowerment an Bedeutung und fanden ihren Ausdruck in der Ausgestaltung der Praxis von Friedensverhandlungen. Auf der anderen Seite zeichnete sich auf der substanziellen Ebene ein „social turn“ (Lee et al. 2016, S. 494) ab, der von einem erweiterten Verständnis von Konfliktursachen getragen war, das - wie oben angedeutet - auch die Frage der patriarchalen Geschlechterverhältnisse einschloss. Das Mandat des peacebuilding begann sich nun konzeptionell auch auf ,nonsecurity issues" (ebda.) zu erstrecken, um einen nachhaltigen und dauerhaften Frieden zu ermöglichen. Im guatemaltekischen Fall waren dies insbesondere die Verhandlungen über Demokratisierung und Menschenrechte, die Rechte und Identität indigener Völker, sozioökonomische Aspekte und die Agrarfrage (Oettler 2004, S. 78).

Einen Meilenstein in der transnationalen Normentwicklung stellte die 2000 verabschiedete Resolution 1325 des UN-Sicherheitsrates dar, die insbesondere auf der prozeduralen Ebene einen Sprung nach vorne bedeutete. Die Resolution unterstrich vor allem die Bedeutung der Partizipation von Frauen in Friedensverhandlungen und Friedensmissionen. Auf der substanziellen Ebene bedeutete die „Gender-Per-

\footnotetext{
Dreh- und Angelpunkt der globalen Peace and Conflict Studies ist noch die AFK das zentrale Forum feministischer Debatten um patriarchale Gewalt- und Geschlechterverhältnisse darstellt, ist der Zeitpunkt des genannten AFK-Kolloquiums geeignet, um eine generelle Trendwende internationaler Debatten anzuzeigen.
} 
spektive“ die Berücksichtigung der Bedürfnisse von Frauen und Mädchen ${ }^{4}$ sowie die Prävention und Aufarbeitung von sexueller Gewalt. In der 2008 verabschiedeten Resolution 1820 des UN-Sicherheitsrates, in der es um den Tatbestand der als Kriegstaktik eingesetzten sexualisierten Gewalt geht, wurde die Bedeutung der Partizipation von Frauen in Peacebuilding, Konfliktprävention und Konfliktbeilegung nochmals unterstrichen. Obwohl diese Dokumente aus queerfeministischer Sicht auch stark kritisiert wurden, da sich in ihnen letztlich ein binäres Geschlechterverständnis spiegelt (Hagen 2016), stellen sie doch wichtige Instrumente dar, derer sich soziale Bewegungen und lokale NGOs in ihren Kämpfen um Frauenrechte und/oder feministische Friedenspolitik bedienen können. Resolution 1325 war insbesondere auch im kolumbianischen Fall ein wesentlicher Kristallisationspunkt für grenzübergreifende Diskursallianzen.

\section{Eine kurze Geschichte des kolumbianischen Friedensprozesses aus feministischer Perspektive}

In den vergangenen Jahrzehnten haben in Kolumbien feministische Mobilisierungen die verschiedenen Bemühungen, bewaffnete Konflikte auf dem Verhandlungsweg zu beenden, begleitet. Die 1982 gegründete Casa de la Mujer (dt. Haus der Frau) war die erste Frauenorganisation, die eine feministische Friedensagenda erarbeitete und Räume schuf, aus denen heraus sich andere Organisationen gründeten.

Die Geschichte der Friedensverhandlungen und der feministischen Friedensbewegung ist indes keine lineare, sondern eine von Fort- und Rückschritten geprägte Pendelbewegung. Auf einen 1984 unter Präsident Belisario Betancur vereinbarten Waffenstillstand und die Gründung der linken Partei Unión Patriótica (UP, dt. Patriotische Union) folgte eine Phase der Repression, der mehrere Tausend Parteimitglieder zum Opfer fielen. ${ }^{5}$ In den frühen 1990er-Jahren wurden unter Präsident César Gaviria zwar keine erfolgreichen Verhandlungen mit der FARC-EP geführt, aber mit einer Reihe kleinerer Guerillagruppen, die sich demobilisierten (Chernick 1999; Jaramillo Paz und Valencia Agudelo 2015). Obwohl diese Verhandlungsprozesse ei-

\footnotetext{
4 Im hier untersuchten Friedensabkommen wird - vermittelt über die Forderungskataloge von Frauenorganisationen - etwa Abs. 8. Der Resolution klar aufgegriffen: ,fordert alle beteiligten Akteure auf, bei der Aushandlung und Umsetzung von Friedensübereinkünften eine Geschlechterperspektive zu berücksichtigen, die unter anderem auf Folgendes abstellt: a) die besonderen Bedürfnisse von Frauen und Mädchen während der Rückführung und Neuansiedlung sowie bei der Normalisierung, der Wiedereingliederung und dem Wieder-aufbau nach Konflikten; b) Maßnahmen zur Unterstützung lokaler Friedensinitiativen von Frauen und autochthoner Konfliktbeilegungsprozesse sowie zur Beteiligung von Frauen an allen Mechanismen zur Umsetzung der Friedensübereinkünfte; c) Maßnahmen zur Gewährleistung des Schutzes und der Achtung der Menschenrechte von Frauen und Mädchen, insbesondere im Zusammenhang mit der Verfassung, dem Wahlsystem, der Polizei und der rechtsprechenden Gewalt“ (UN Sicherheitsrat 2000, S. 194).

5 Dieser Fall wird derzeit als „Fall 006“ von der JEP (Justicia Especial para la Paz, Spezialgerichtsbarkeit für den Frieden) untersucht. Das Centro Nacional de Memoria Histórica dokumentiert in dem Bericht ,Todo pasó frente a nuestros ojos. El genocidio de la Unión Patriótica 1984-2002“ 4153 Todesopfer (CNMH 2018, S. 108). Dem CNMH wird unter seinem neuen Direktor Dario Acevedo ein Rechtsruck vorgeworfen, so verwundert es nicht, dass der link zu dem Bericht auf der Seite des CNMH nicht mehr zu funktionieren scheint. Der Bericht ist auch auf der Seite der JEP zugänglich.
} 
ne genderbezogene Leerstelle aufwiesen (Chaparro González und Martínez Osorio 2016, S. 37), wurde mit der Erarbeitung und Verabschiedung einer neuen Verfassung 1991 eine neue Ära im demokratischen Selbstverständnis Kolumbiens eingeleitet. In der Verfassung von 1991 drückte sich nicht nur die Stärkung der Menschenrechte und der politischen Partizpationsmöglichkeiten der Bevölkerung aus, sondern auch eine allmähliche Aufweichung traditioneller katholischer Familienformen. Die politische Realität der 1990er-Jahre war indes vor dem Hintergrund des grassierenden Paramilitarismus und der florierenden Drogenökonomie weit vom demokratischen Idealzustand entfernt und die Präsidentschaft von Ernesto Samper (1994-1998) war von dem Vorwurf der Korruption und Annahme von Drogengeldern überschattet.

Im Kontext extremer Manifestationen von paramilitärischer, krimineller, aufständischer und staatlicher Gewalt gründete sich 1996 die Ruta Pacífica de las Mujeres (dt.: friedlicher Weg der Frauen), um in nationalen und internationalen Diskursräumen Aufmerksamkeit für das Phänomen der systematisch ausgeübten sexuellen/ sexualisierten und gender-basierten Gewalt zu schaffen und eine gendersensible Agenda für den Frieden durchzusetzen. Das erste medial wirksame Ereignis war ein Demonstrationszug nach Urabá (Antioquia), an dem sich 1500 Frauen beteiligten. ${ }^{6}$ Die Gründungsdeklaration der Ruta Pacífica vom 25. November 1996 unterstrich die Notwenigkeit regionaler Dialoge, lokaler Entwicklungen und fundamentaler Änderungen von Mentalitäten und Haltungen (Villarraga Sarmiento 2013, S. 301-302). Damit hatte die Ruta Pacífica, die sich schnell zu einem der wichtigsten Dachverbände von Frauen- und feministischen Organisationen entwickeln sollte, bereits in den 1990er-Jahren eine inklusive Friedensagenda entworfen, die auch die Fragen der politischen Partizipation und Konfliktursachen adressierte. Hier zeigt sich also deutlich, dass es sich um einen ko-konstitutiven Prozess der Normentwicklung handelte, der verschiedene nationale und lokale Akteurinnen zusammenbrachte, die zugleich auch Teil internationaler feministischer Bewegungen waren.

Unter Andrés Pastrana (1998-2002) kehrten FARC-EP und Regierung zu einer breiten Verhandlungsagenda zurück. Der Verhandlungsprozess, der von zahlreichen zivilgesellschaftlichen Aktivitäten und Initiativen begleitet wurde (Zulaga Nieto, 2013, p. 49), konzentrierte sich geographisch auf Caguán - ein entmilitarisiertes Gebiet von der Größe der Schweiz im Süden des Landes. In diesem Verhandlungsprozess zeigte sich auch deutlich die im vorangegangenen Abschnitt skizzierte internationale Normentwicklung der 1990er-Jahre, die auf eine nachhaltige Friedenskonsolidierung durch die Stärkung von Menschenrechten und Rechtsstaatlichkeit sowie durch soziale und marktwirtschaftliche Reformen ausgerichtet war. Da dies auf der prozeduralen Ebene die Einführung von partizipativen Mechanismen auch auf der Ebene der Friedensverhandlungen beinhaltete, einigten sich die kolumbianischen Verhandlungsparteien auf die Durchführung von ,thematischen Foren“ in San Vicente de Caguán. Während die Verhandlungen keine greifbaren Erfolge zeitigten und letztlich 2002 scheiterten, läutete der zwischen Pastrana und der US-Regierung vereinbarte Plan Colombia eine politische Kehrtwende ein, die unter Álvaro Uribe (2002-2010), der auf den militärischen Sieg gegen aufständische Gruppierungen setzte, die Gestalt der ,demokratischen Sicherheit“ annahm.

\footnotetext{
${ }^{6}$ https://rutapacifica.org.co/wp/ruta-pacifica-regional-antioquia/.
} 
Für die Geschichte des kolumbianischen Friedensprozesses stellen die Verhandlungen während der Amtszeit von Pastrana einen wichtigen Meilenstein dar, weil sich eine inklusive Friedensagenda sowohl auf der substanziellen als auch auf der prozeduralen Ebene konkretisierte. Zwar hatte es diesbezüglich - wie oben beschrieben - erste Ansätze bereits unter Betancur gegeben (vgl. auch Kurtenbach 2001), doch wurde nun sowohl die Partizipation zivilgesellschaftlicher Akteure als auch die thematische Breite der Verhandlungsagenda erstmals konkretisiert. Aus der Sicht von feministischen Bewegungen war dies zugleich eine Zeit der schnellen Akkumulation von Handlungserfahrungen, Deutungsmustern und Strategien der Ressourcenmobilisierung. Das Spektrum feministischer Organisationen und Netzwerke weitete sich stetig aus und repräsentierte eine Vielfalt von sozialen Positionen sowie politischen Diskursen (Villarraga Sarmiento 2013). Viele NGOs und Gruppierungen stützten ihre Arbeit auf eine breite und akademische feministische Agenda, andere konzentrierten sich auf spezifische Problemfelder und wiederum andere engagierten sich im Bereich von lokaler Friedensförderung und female leadership (Rojas 2009).

1998 wurde die Confluencia Nacional de Redes de Mujeres als ein in alle Regionen des Landes ausgreifendes Metanetzwerk gegründet. Insgesamt zeichnet sich das Spektrum der kolumbianischen Frauenbewegung durch Multiperspektivität, Diskursstärke und organisatorische Kraft aus. Das 1991 gegründete Netzwerk Red Nacional de Mujeres (dt. Nationales Frauennetz), die bereits genannte Ruta Pacífica und die 2001 ins Leben gerufene Iniciativa de Mujeres por la Paz (dt.: Fraueninitiative für den Frieden) zählten zu den wichtigsten feministischen Akteurinnen im Feld der kolumbianischen Friedenspolitik. In den genannten Netzwerken organisierten sich unterschiedliche Gruppen und brachten komplementäre - und zum Teil auch konfliktive - kleinbäuerliche, akademische, afro-kolumbianische, indigene und christliche Perspektiven in die Debatten ein (Rojas 2009; Quintero 2003). In den späten 1990er und frühen 2000er Jahren engagierte sich die Frauenbewegung stark im Kontext der Friedensverhandlungen und trug auch durch die Vermittlung von lokalen Friedensabkommen (Bouvier 2016, S. 18) zum Friedensprozess bei. Die Beteiligung an thematischen Foren und die Erarbeitung von Forderungskatalogen führte indes noch nicht dazu, dass diese auch in den offiziellen Verlautbarungen der Konfliktparteien Berücksichtigung fanden (Chaparro González und Martínez Osorio 2016, S. 55). In den 1990er-Jahren begann ein allmählicher Aufschwung der LGBT-Bewegung, die nach ihrer frühen libertären Phase in den 1980er-Jahren aufgrund von paramilitärischen ,sozialen Säuberungen“ und den Auswirkungen der HIV/Aids-Pandemie kaum organisations- und handlungsfähig war. Aus der Perspektive der LGBT-Bewegung stellte die Initiative Planeta Paz (dt.: Planet Frieden) einen entscheidenden Meilenstein dar, da sie 2000 erstmals LGBT-Aktivist*innen aus verschiedenen Landesteilen im Kontext der Debatte über Friedensinitiativen zusammenbrachte (Oettler 2019). Die Erfahrungen und Forderungen der LGBT-Bewegung begannen nun, auch in der Friedensagenda von Frauen- und feministischen Bewegungen sichtbarer zu werden.

Der Amtsantritt von Álvaro Uribe (2002-2010), der auf einen militärischen Sieg über die Guerilla setzte, markierte einerseits einen deutlichen Einschnitt in der Geschichte des kolumbianischen Friedensprozesses, da er nicht mit der FARC-EP in substanzielle Verhandlungen einzutreten bereit war. Dies sollte sich erst unter seinem 
Nachfolger Manuel Santos (2010-2017) ändern. Andererseits legten beide Regierungen die Fundamente einer opferzentrierten Transitional-Justice-Architektur. Die zentralen gesetzlichen Meilensteine - die Gesetze 975 und 1448 aus den Jahren 2005 und 2011 - verdankten sich dabei einem erfolgreichen Verhandlungsprozess mit dem paramilitärischen Dachverband AUC (Autodefensas Unidas de Colombia, dt: Verinigte Bürgerwehren Kolumbiens), der zur Demobilisierung von 30.000 Paramilitärs führte (Tate 2009).

Während sich die humanitäre Krise zuzuspitzen begann, versuchte die feministische Bewegung, nationale und internationale Aufmerksamkeit für die geschlechtsspezifischen Folgen von systematischen Vertreibungen zu erzeugen. Zu den zentralen Forderungen gehörten die (Wieder-)Aufnahme von Friedensverhandlungen sowie die Demilitarisierung des zivilen Lebens. Damit verbunden war eine Fülle von sozialen Problemlagen und spezifischen Konfliktursachen. Noch 2002 - diesmal organisiert von der Iniciativa de Mujeres por la Paz - wurde eine Constituyente Emancipatoria de Mujeres (dt.: Emanzipatorische verfassungsgebende Versammlung der Frauen) veranstaltet, um eine neue feministische Aktionslinie $\mathrm{zu}$ formulieren. Ein erstes Dokument mit 600 Stichpunkten, die 700 Repräsentant*innen zusammengetragen hatten, wurde im Laufe der Diskussionen in ein kürzeres Format gebracht (Rojas 2009, S. 212).

\section{Die Verhandlungen in Havanna und das Plebiszit}

Diese Diskussionen fanden zehn Jahre später endgültig Eingang in den formalen Friedensprozess, als Präsident Santos im November 2012 in Havanna mit Vertreter*innen der FARC-EP Vorverhandlungen aufnahm. Nachdem sich abzeichnete, dass keine Frauen in den Verhandlungsdelegationen repräsentiert sein würden, riefen zivilgesellschaftliche Akteur*innen abermals ein Metanetzwerk - Mujeres por la Paz (dt.: Frauen für den Frieden) - ins Leben, um die Umsetzung der Richtlinien der Resolution 1325 des UN-Sicherheitsrates und damit die Partizipation von Frauen und die Einbeziehung einer Genderperspektive einzufordern (Céspedes-Baéz/Jaramillo Ruiz 2018, S. 92-95, Chaparro González und Martínez Osorio 2016, S. 68-72). Von den vielfältigen öffentlichen Aktivitäten war sicherlich das dreitägige Treffen von Frauenorganisationen und Netzwerken im Oktober 2013 am Wichtigsten. An diesem Cumbre Nacional de Mujeres por la Paz (dt: Nationales Gipfeltreffen von Frauen für den Frieden) nahmen 450 Frauen teil und trugen unterschiedliche lokale, nationale und internationale Erfahrungen zusammen. In insgesamt 12 Arbeitsgruppen wurde eine sehr umfangreiche und detaillierte Liste von konkreten Vorschlägen für den Verhandlungsprozess und die Verhandlungsagenda erarbeitet (Cumbre Nacional de Mujeres 2014, S. 74-91). Die Forderungen von kolumbianischen Frauen- und feministischen Organisationen erhielten durch die Unterstützung von internationalen Organisationen, insbesondere UN Women, sowie norwegischen Diplomat*innen ein entscheidendes zusätzliches Gewicht (Salvesen und Nylander 2017). Im November 2013 ernannte die Regierung Nigeria Rentería und María Paulina Riveros zu Mitgliedern ihrer Verhandlungsdelegation. Der starke nationale und internationale Druck führte schließlich zur Einrichtung einer Gender-Unterkommission, deren zentrale 
Funktion in der Mitwirkung bei der Erstellung und Überarbeitung der genderbezogenen Bestimmungen des Friedensvertrages bestand (Presidencia de la República, Oficina del Alto Comisionado para la Paz 2018). Die Verhandlungspartner führten 2014 in Havanna insgesamt fünf Hearings mit 60 Repräsentant*innen von Opferorganisationen durch, von denen 36 Frauen waren. Darüber hinaus traf sich die Gender-Subkommission mit einer Reihe von kolumbianischen Aktivist*innen, Repräsentant*innen internationaler Organisationen und demobilisierten Guerilleras aus anderen Ländern. Während die Mitglieder der Gender-Unterkommission breite Unterstützung durch feministische Expert*innnen und Aktivist*innen erfuhren, wurde ihre Aufgabe innerhalb der männlich geprägten Verhandlungsdelegationen als untergeordnete „Frauenangelegenheit“ wahrgenommen. Die Mitglieder der GenderUnterkommission mussten ,,sich noch etwas mehr opfern“7 und zusätzliche Arbeit leisten. Die Gender-Unterkommission hatte eine starke Bedeutung für die Überarbeitung und endgültige Ausformulierung der gender-bezogenen Bestimmungen der Kapitel zur Landreform, politischen Partizipation und Drogenproblematik (s. nächster Abschnitt). Aufgrund der jahrelangen Lobbyarbeit von Frauen-, LGBT- und feministischen Bewegungen trug der Friedensvertrag, der der kolumbianischen Bevölkerung im Oktober 2016 zur Abstimmung vorgelegt wurde, deutlich die Handschrift einer inklusiven und feministischen Friedenspolitik.

In den Öffentlichkeitskampagnen, die im Vorfeld des Plebiszits geführt wurden, spiegelte sich die Zerrissenheit einer höchst unterschiedlich von den bewaffneten Konflikten der vergangenen Jahrzehnte betroffenen Gesellschaft. In den Kampagnen gegen das Friedensabkommen, die prominent in sozialen Medien geführt wurden, spielte einerseits das Motiv einer vermeintlichen Kapitulation vor dem Terrorismus sowie die Ablehnung von Transitional Justice und der garantierten Repräsentation der FARC in den beiden Kammern des Parlaments in den nächsten zwei Legislaturperioden eine Rolle. Es ging ferner um die immensen Kosten eines Friedensprozesses (Opferentschädigungen, Sozialprogramme, Landverteilung und Reintegrationsprogramme). Die Stimmung wurde zusätzlich durch die Verbreitung von fake news geschürt. Dazu gehörte der Hinweis auf Rentenkürzungen (um Reintegrationsprogramme zu finanzieren) ebenso wie das Gespenst eines drohenden „Castrochavismus" unter einem künftigen FARC-Präsidenten. Eine besondere Rolle spielte in diesen Kampagnen der Vorwurf der atheistischen „Gender-Ideologie“. Die Dynamik, die sich diesbezüglich entwickelte, stand in der Sogwirkung von scharfen sexualpolitischen Auseinandersetzungen. Mitte 2016 erhitze eine Kampagne gegen die offen lesbische Bildungsministerin Gina Parody die Gemüter. Anlass war eine Broschüre zur Sexualerziehung, mit der das Bildungsministerium - unterstützt von internationalen Organisationen und der kolumbianischen NGO Colombia Diversa auf ein Urteil des Obersten Gerichts aus dem Jahre 2015 reagierte. Dieses hatte nach dem Selbstmord des Schülers Sergio Urrego eine diskriminierungssensible Sexualerziehung an Schulen eingefordert (Castillo Vargas 2019, S. 116). Im August 2016 mobilisierten rechte Politiker*innen und Religionsvertreter*innen erfolgreich zu Demonstrationen in Bucaramanga, Barranquilla, Bogotá und anderen Städten. Die zum

\footnotetext{
${ }^{7}$ Interview with member of the subcommission, in: Corporación Humana/CIASE, 2017, p. 29, see also p. 43.
} 
Teil auf fake news beruhende Kampagne wurde im Kontext der Debatte über das Friedensabkommen fortgesetzt. Dabei wurde - ganz abgekoppelt vom eigentlichen Vertragstext - vor der ,homosexuellen Agenda“, Abtreibung und der Zerstörung der traditionellen Familie gewarnt (s. ausführlich Esguerra Muelle 2017). Der wesentliche Beleg für diese Vorwürfe war die häufige Verwendung der Begriffe ,gender“ und „LGBT“ im Vertragstext. In einer stark polarisierten und aufgeheizten Debatte wurde ausgerechnet die Inklusivität des Friedensvertrages einer der Gründe für sein vorläufiges Scheitern.

\section{Die Folgen der Debatte um „Genderideologie“: Zwei Versionen des Friedensvertrages}

Auf das gescheiterte Plebiszit am 02.10.2016 folgte ein kurzer und intensiver Revisionsprozess von 41 Tagen. Präsident Santos berichtete, 500 Änderungsvorschläge erhalten zu haben, die in 57 Themenfelder gruppiert und von den Verhandlungsdelegationen „Punkt für Punkt“" bearbeitet worden seien. ${ }^{8}$ Das Ergebnis war ein modifiziertes Dokument, das schließlich am 24.11.2016 im hauptstädtischen Theater Colón in einer eher unspektakulären Zeremonie unterzeichnet wurde. Bei dem modifizierten Friedensabkommen handelte es sich um keinen grundsätzlichen Neuentwurf, sondern um ein Dokument, das zwar in einigen zentralen Punkten Zugeständnisse an das „No“-Lager machte, aber vor allem eine Fülle von Einzeländerungen enthielt. Zu den Kernpunkten zählten die Rücknahme des verfassungsrechtlichen Status des Friedensvertrages, die Präzisierung (und Begrenzung) der Laufzeit der Übergangsjustiz (JEP) auf 10 Jahre (mit einer Verlängerungsoption von 5 Jahren), der Verzicht auf ausländische Richter*innen der JEP, die Offenlegung der Finanzen der FARCEP und ihrer Verbindung zum Drogenhandel sowie die zeitliche Ausdehnung der vorgesehenen Landreform auf 15 Jahre.

Der Vergleich der beiden Versionen des Friedensvertrages vom 24.08.2016 und 12.11.2016 ${ }^{9}$ zeigt, dass der Umgang mit dem kontroversen Thema ,gender“ ambivalent ist, da zwar auf die vehemente Kritik an einer aufoktroyierten „GenderIdeologie“" reagiert wird, aber doch auch einige Präzisierungen und Erweiterungen vorgenommen werden. Eine einfache Wortzählung zeigt die Richtung der Überarbeitungen an. Während im Vertragstext vom 24.08.2016 die Begriffe ,género“, „mujeres“ und „LGBTI“ 114x, 185x und 10x vorkommen, tauchen diese Begriffe (in der gleichen Reihenfolge) im modifizierten Friedensvertrag 55x, 209x und 16x auf. Dies lässt darauf schließen, dass es vor allem darum ging, den umstrittenen gender-Begriff zu vermeiden, um die Kritikanfälligkeit des Dokumentes zu reduzieren. Tatsächlich finden sich viele Stellen, an denen der Begriff ,género“ ersatzlos

\footnotetext{
8 Restrepo, Vanesa: 57 propuestas de voceros del No serán discutidas con las Farc. El Colombiano, 05.11 .2020 .

https://www.elcolombiano.com/colombia/acuerdos-de-gobierno-y-farc/gobierno-y-farc-discutiranpropuestas-entregadas-por-voceros-del-no-DA5310511 (Zugriff: 06.03.2020).

9 Der Vergleich wird durch dieses tool wesentlich erleichtert: https://draftable.com/compare/Jjyp TOknafBktqve (Zugriff 06.03.2020). Die folgenden Verweise beziehen sich auf die plebiszitäre (AI) und postplebiszitäre (AII) Fassung des Friedensabkommens, Gobierno Nacional, und FARC-EP 2016a, b.
} 
gestrichen oder durch unverfänglichere Wendungen ersetzt wurde. Die ,equidad de género“ (Gendergerechtigkeit, AI, S. 9) wurde zur ,,igualdad de oportunidades entre hombres y mujeres" (Chancengleichheit zwischen Männern und Frauen, AII, S. 10); aus den „personas con orientación sexual e identidad de género diversa“ (Personen mit abweichender sexueller Orientierung und gender-Identität, AI, S. 21) wurden „grupos en condición de vulnerabilidad“ (vulnerable Gruppen, AII, S. 25), der ,enfoque diferencial y de género" (Differenz- und gender-Fokus, AI, S. 27) wurde auf den Nenner der ,situación particular de las mujeres“ (besondere Situation der Frauen, AII, S. 31) gebracht, die „valores no sexistas“ (nicht-sexistischen Werte, AI, S. 40) wurden in ,valores no discriminatorios" (nicht-diskriminierende Werte, A II, S. 46) umgedeutet, und der ,enfoque de género“ (Gender-Fokus, AI, S. 124) wurde auf „este enfoque“ (diesen Fokus, AII, S. 137) verkürzt. Diese Beispiel zeigen an, dass es bei der Revision des Vertragstextes darum ging, einen Begriff zu umgehen, der ein Verständnis von Geschlecht als biologisch gegebener Eigenschaft ablehnt und vielmehr jene sozialen Prozesse betont, die Geschlecht als folgenreiche soziale Unterscheidung produziert und reproduziert. Insofern sind jene Stellen emblematisch, in denen z. B. bezüglich der Auswahl der Mitglieder der Wahrheitskommission aus der Berücksichtigung der ,equidad de género“ (Gendergerechtigkeit, AI, S. 125) die „participación equitativa entre hombres y mujeres“ (gleichmäßige Beteiligung von Männern und Frauen, A II, S. 137) gemacht wurde.

Obwohl hier letztlich ein binäres Geschlechtermodell reproduziert wird, sollte dieser diskursive Schwenk nicht überbewertet werden, denn insgesamt ist die Überarbeitung des Dokuments sehr widersprüchlich. Bemerkenswert ist zunächst, dass die Existenz nicht-heteronormativer Identitäten nicht per se negiert wird, so wird etwa an einer Stelle die ,identidad de género diversa“ (abweichende Genderidentität AI, S. 47) als „LGBTI“ (AII, S. 53) übersetzt. Besonders interessant ist zweitens eine Stelle, an der eine diskriminierungssensible Position durch den Überarbeitungsprozess gestärkt wurde. Die ,promoción de la no estigmatización en razón de la orientación sexual y la identidad de género diversa" (Förderung der Nicht-Stigmatisierung aufrund von sexueller Orientierung und abweichender Genderidentität, AI, S. 41) wird auf ,grupos en condición de vulnerabilidad o discriminados como las mujeres, los pueblos y comunidades étnicas, población LGBTI, los jóvenes, niños y niña, adultos mayores, las personas en condición de discapacidad, las minorías políticas y las minorías religiosas" (vulnerable oder diskriminierte Gruppen wie Frauen, indigene Völker und Gemeinden, LGBTI-Personen, Jugendliche, Kinder, Alte, Menschen mit Behinderungen, politische und religiöse Minderheiten, A II, S. 47) erweitert. Während diese Wendung auch die Frage aufwirft, welche politischen und religiösen Minderheiten (evangelikale Kirchen, Judentum, Islam, Rastafarianismus?) gemeint sind, ist zu betonen, dass sich die wesentliche Konzession an das „No“-Lager zu Beginn des Vertragstextes findet.

In der ursprünglichen Einleitung wurde zunächst betont, 1) dass der Vertrag aus einer Reihe von miteinander verbundenen Abkommen bestünde, die von demselben rechtsbasierten Ansatz und dem Wunsch, zur Verwirklichung der verfassungsgemäßen Rechte der Kolumbianer beizutragen, getragen seien. Außerdem seien sie 2) von einem Differenz und Gender-Fokus geleitet, der sicherstellen solle, dass während des Implementationsprozesses der Gender-, ethnischen und kulturellen Pluralität 
Rechnung getragen wird und dass Maßnahmen für die besonders vulnerablen oder benachteiligten Gruppen - insbesondere Kinder, Frauen, Menschen mit Behinderung und Opfer - ergriffen werden und dies auch unter Berücksichtigung unterschiedlicher territorialer Betroffenheit. ${ }^{10}$ In der revidierten Version wird der Gender-Fokus gleichsam zurückgestellt, indem Absatz 2) mit dem Hinweis beginnt, dass das Friedensabkommen ohne jede Ausnahme den Primat der unveräußerlichen Rechte der Menschen als Fundament des Zusammenlebens im Öffentlichen und Privaten anerkenne sowie die Familie als Keimzelle der Gesellschaft und der Rechte ihrer Angehörigen. Es folgt ein Bekenntnis zu Gleichheit und Pluralismus sowie zu affirmativen Maßnahmen für diskriminierte und marginalisierte Gruppen, wobei der Territorial-, Differenz- und Gender-Fokus zum Tragen käme ${ }^{11}$. Die hier ausführlich paraphrasierte Passage zeigt sehr deutlich das Bemühen, den Appellen gegen eine „Genderisierung“ stattzugeben und - insbesondere im Kontext von parallelen Auseinandersetzungen um schulische Sexualerziehung - den Wert der Familie nicht nur zu unterstreichen, sondern ihr auch einen Primat vor individuellen Rechten einzuräumen. Während so also in der Einleitung des Textes eine deutliche Konzession an die Gegner*innen des Friedensabkommens vorgenommen wurde, war die Revision des Vertragstextes zugleich die Möglichkeit, an einigen der zuletzt verhandelten Punkte noch Neujustierungen vorzunehmen, die die Gender-Perspektive eher stärkten als schwächten. Beispiele sind die Benennung von Schweden als weiterem an der Verifikation der Gender-Dimension des Abkommens beteiligtem Akteur oder die Berücksichtigung der Gesundheitssituation gebärender und stillender Mütter während der Demobilisierung der Guerilla.

Was die knappe Diskussion der genderbezogenen Änderungen des Friedensvertrages schon gezeigt hat, ist der Umstand, dass ,gender“ für den Friedensprozess eine doppelte Bedeutung hat. Gender ist einerseits ein Querschnittsthema, das sich - wie auch der Aspekt der territorialen Ungleichheiten - durch den gesamten Vertragstext zieht und programmatisch auf fast alle verhandelten Bereiche ausstrahlt. „Gender" ist damit ein normatives Element und Gendergerechtigkeit ist die Absicht, die hinter dem Friedensprozess als policy steht. Andererseits übersetzt sich die Genderperspektive im Vertragstext selbst in eine Vielzahl von konkreten Maßnahmen auf verschiedenen Ebenen und in verschiedenen Politikfeldern. Dabei geht es - und dies sind die einzelnen Kapitel des Friedensvertrages - um rurale Reformen, po-

\footnotetext{
${ }^{10}$ El Acuerdo está compuesto de una serie de acuerdos, que sin embargo constituyen un todo indisoluble,porque están permeados por un mismo enfoque de derechos, para que las medidas aquí acordadas contribuyan a la materialización de los derechos constitucionales de los colombianos; por un mismo enfoque diferencial y de género, para asegurar que la implementación se haga teniendo en cuenta la diversidad de género, étnica y cultural, y que se adopten medidas para las poblaciones y los colectivos más humildes y más vulnerables, en especial los niños y las niñas, las mujeres, las personas en condición de discapacidad y las víctimas; y en especial por un mismo enfoque territorial. (AI: 4).

${ }^{11}$ El Acuerdo Final reconoce, sin discriminación alguna, la primacía de los derechos inalienables de la persona como fundamento para la convivencia en el ámbito público y privado, y a la familia como núcleo fundamental de la sociedad y los derechos de sus integrantes. La implementación del Acuerdo deberá regirse por el reconocimiento de la igualdad y protección del pluralismo de la sociedad colombiana, sin ninguna discriminación. En la implementación se garantizarán las condiciones para que la igualdad sea real y efectiva y se adoptarán medidas afirmativas en favor de grupos discriminados o marginados, teniendo en cuenta el enfoque territorial, diferencial y de género. (AII: 6).
} 
litische Partizipation, Drogenökonomie, Transitional Justice, Demobilisierung und Wiedereingliederung der Guerilla und die Implementation des Friedensvertrages.

\section{Gender im Friedensvertrag: 310 Seiten, 100 Bestimmungen}

Der Friedensvertrag zwischen der kolumbianischen Regierung und der FARC-EP ist ein komplexes Abkommen, das in einen ebenso komplexen Kontext lokaler, regionaler, nationaler und internationaler Institutionen eingebettet ist. Der im November 2016 unterzeichnete Vertrag umfasst 310 Seiten, auf denen die Begriffe „Gender“, „Frauen“ und „LGBTI“ 280 Mal vorkommen. Während ONU Mujeres (2018) 100 gender-bezogene Maßnahmen identifiziert, die im Friedensvertrag vorgesehen sind, enthält der von der kolumbianischen Regierung erarbeitete und von der Comisión de Seguimiento, Impulso y Verificación del Acuerdo Final (CSIVI) verabschiedete Plan Marco de Implementación (2016) insgesamt 50 Strategien und Indikatoren zum Gender-Fokus des Friedensvertrages.

Wie lässt sich eine analytische Schneise in das Dickicht der Bestimmungen des Vertrages schlagen? Im Folgenden wird zwischen verschiedenen Qualitätsdimensionen unterschieden und damit eine Strukturierung gender-bezogenen Bestimmungen des Vertrags vorgenommen. Dabei sind zwei Qualitätsdimensionen von besonderer Bedeutung. Erstens wird nach der Art der Konfliktbezogenheit unterschieden: 1) Konfliktursachen, 2) Konfliktfolgen und 3) Konfliktbeilegung. Zweitens wird nach dem Konkretisierungsgrad der einzelnen Bestimmungen im Friedensvertrag unterschieden: 1) Leitbilder und prioritäre Handlungsfelder, 2) Repräsentation von Akteur*innen, 3) konkrete Projekte und Maßnahmen, 4) Schaffung von Institutionen.

Auf der Grundlage der von ONU Mujeres (2018) vorgenommenen Identifikation von etwas mehr als 100 genderbezogenen Maßnahmen ergibt sich folgendes Bild, das sicherlich nicht ganz trennscharf ist, denn einerseits fächern sich einige diese Maßnahmen in unterschiedliche Zielvorgaben auf ${ }^{12}$ und andererseits ist oft nicht klar zu unterschieden, ob eine Maßnahme Leitbild/Handlungsfeld oder bereits ein kon-

Tab. 1 Qualitätsdimensionen des Genderfokus im Friedensvertrag zwischen Regierung und FARC-EP (2016)

\begin{tabular}{lrrrr}
\hline & \multicolumn{2}{c}{ Gegenstand der Maßnahme } & & \\
& Konfliktursachen & Konfliktfolgen & Konfliktbeilegung & Summe \\
\hline Leitbild/Handlungsfeld & 30 & 9 & 7 & 46 \\
Partizipation/ & 17 & 8 & 2 & 27 \\
Repräsentation & 18 & & 5 & 26 \\
Projekt & 2 & 3 & 2 & 8 \\
Institution & 67 & 4 & 16 & 107 \\
Summe & 24 & & \\
\hline
\end{tabular}

Quelle: eigene Darstellung auf Basis von ONU Mujeres (2018)

12 Das Kroc Institute (2019) zählt insgesamt 578 Maßnahmen, von denen 130 einen Gender-Bezug aufweisen würden. 
kretes Projekt ist. Gleichwohl ergibt sich aus einer nicht immer scharfen Zuordnung ein aufschlussreiches Bild. (Tab. 1).

Insgesamt stellt sich heraus, dass es ein starkes Gewicht jener Bestimmungen gibt, die ein geringes Niveau an Konkretisierung aufweisen. So sind etwa „die Unterstützung der Bildung und Stärkung von Frauen- und Jugendorganisationen sowie von historisch diskriminierten Gruppen durch außerordentliche Maßnahmen der rechtlichen und technischen Unterstützung“ oder die „Förderung der Ausweitung der gleichberechtigten Partizipation von Frauen in Partizpationsinstanzen“ recht vage Formulierungen (ONU Mujeres 2018, S. 19). Es werden hier Leitbilder, prioritäre Handlungsfelder und auch konkrete Maßnahmen genannt, aber keine Indikatoren der Zielerreichung definiert. Das Grundverständnis der Genderperspektive als Querschnittsthema bedeutet auch, dass nur wenige konkrete Institutionen geschaffen werden sollen, dazu gehören neben einer dem Innenministerium zugeordneten Kommission zur Unterstützung der Aufarbeitung von Verbrechen an Mitgliedern von sozialen Bewegungen (ONU Mujeres 2018, S. 18) ${ }^{13}$ und konkreten Arbeitsgruppen innnerhalb des Transitional-Justice-Systems vor allem die 2017 gegründete Instancia de Alto Nivel de Gobierno para la Incorporación del Enfoque de Género (dt.: Hochrangige Regierungsinstanz zur Einbeziehung des Gender-Fokus).

Neben der Konkretisierung der Maßnahmen ist die Frage zentral, worauf sich die verschiedenen genderbezogenen Maßnahmen beziehen. Laut ONU Mujeres (2018) verteilen sie sich wie folgt auf die Kapitel des Friedensvertrags: Kapitel I (Integrale Landreform): 27, Kapitel II (Politische Partizipation): 17, Kapitel III (Ende des Konfliktes): 16; Kapitel IV (Drogen): 17, Kapitel V (Opfer): 15, und Kapitel VI (Implementation): 8.

Wesentlich aufschlussreicher noch als diese Aufschlüsselung ist indes die Frage, ob sich die genderbezogenen Maßnahmen auf die Bearbeitung von Konfliktursachen oder Konfliktfolgen oder auf die Konfliktregelung beziehen. Dem Begriff der Konfliktregelung werden hier jene Maßnahmen zugeordnet, die sich unmittelbar auf die Beendigung des bewaffneten Konflikts zwischen staatlichen Kräften und FARC-EP beziehen. Dazu zählen die Modalitäten der Entwaffnung und Demobilisierung der Guerilla ebenso wie die Implementierung, Verifizierung und internationale Begleitung des Friedensprozesses. Zwar ist die Anzahl der genderbezogenen Maßnahmen in diesem Bereich geringer als in den anderen beiden Bereichen, aber dennoch handelt es sich hier um sehr relevante Maßnahmen. Neben einer Reihe von konkreten gender-bezogenen Maßnahmen im Kontext der Demobilisierung und Reinkorporation der FARC-EP-Kämpfer*innen geht es hier insbesondere um die Verankerung von Gender als prioritärem Handlungsfeld im Plan Marco (dt. Rahmenplan) zur Umsetzung des Abkommens sowie um die Repräsentation von Frauen- und LGBTIOrganisationen im Prozess der Umsetzung des Friedensvertrages. Von besonderer Bedeutung ist diesbezüglich die Bestimmung zur Einrichtung und Besetzung der Instancia Especial de Mujeres (dt: Spezialinstanz der Frauen).

Die gender-bezogenen Maßnahmen zum Umgang mit den Konfliktfolgen finden sich vor allem im fünften Kapitel des Friedensvertrages, in dem die Transitional-

13 Comité de impulso a las investigaciones por delitos contra líderes y lideresas sociales y defensores y defensoras de derechos humanos. 
Justice-Architektur festgelegt wird, sowie im dritten Kapitel in den Bestimmungen zum Umgang mit paramilitärischen Strukturen. Auch dieser Bereich zeichnet sich dadurch aus, dass genderbezogene Maßnahmen auf allen Konkretisierungsebenen formuliert werden. Es wird insbesondere festgelegt, dass die Transitional-JusticeInstitutionen den Auftrag erhalten, gender-bezogene Gewalt zu adressieren, Frauen und andere benachteiligte Gruppen angemessen $\mathrm{zu}$ repräsentieren und spezifische Arbeitsgruppen zur Bearbeitung dieser Thematik zu schaffen.

Es ist mehr als nur bemerkenswert, dass sich die überwiegende Mehrzahl der genderbezogenen Maßnahmen auf Ansätze zur Lösung struktureller Konfliktursachen beziehen. Diesbezüglich ist der Konkretisierungsgrad relativ gering, es sollen mit der bereits genannten Kommission zur Unterstützung der Aufarbeitung von Verbrechen an Mitgliedern von sozialen Bewegungen und einem Integralen Nationalen Interventionsprogramm gegen den Drogenkonsum lediglich zwei stärker institutionalisierte Strukturen geschaffen werden. Im Feld der konfliktursachenbezogenen Maßnahmen findet sich eine Vielfalt an Einzelaspekten wie z. B. spezielle Kreditlinien für Frauen, Gesundheitsförderung und HIV-Prävention für Gefängnisinsassinnen oder das Gebot nicht-diskriminierender Inhalte in staatlichen Kommunikationsmedien. Viele dieser Vereinbarungen haben einen verbindlichen Leitbildcharakter, ohne dass jedoch Indikatoren oder Erfolgskriterien festgelegt werden. Was bedeutet z. B. die „Sichtbarmachung der Arbeit von lideresas sociales und Menschenrechtsaktivistinnen“ (ONU Mujeres 2018, S. 18) konkret? Wann ist die „Implementierung von Maßnahmen zur Armutsbekämpfung, die auf die Bedürfnisse von Frauen reagieren und die Chancengleichheit von Männern und Frauen erreichen“ (ONU Mujeres 2018, S. 14) erfolgreich? Was genau bedeutet es, einen Gender- und Differenzfokus in den Programmlinien von Bildungs- oder Gesundheitsministerien zu verankern? Insgesamt besteht ein Hauptproblem sicherlich in der Unübersichtlichkeit und mangelnden Operationalisierung der genderbezogenen Bestimmungen.

Im Hinblick auf Agenda-Setting, Politikformulierung und Politikdurchführung stellt sich hier auch die doppelte Frage der Legitimität und Verantwortlichkeit. Der Friedensvertrag skizziert ein fast unüberschaubares Geflecht an Verantwortlichkeiten, das von verschiedenen Ministerien bis hin zur Nationalen Landagentur (Agencia Nacional de Tierras), der Agrarbank (Banco Agrario), dem Geographieinstitut Augustín Cordazzi, dem Nationalen Wahlrat, einer Reihe von präsidialen Planungsbehörden, der Rundfunkanstalt sowie Bürgermeister*innen und den im Zuge des Friedensprozesses geschaffenen lokalen, nationalen und internationalen Institutionen reicht. Das Panorama der verantwortlichen Akteure verweist auf ein grundsätzliches Legitimitätsproblem, denn der Friedensvertrag sieht ein ganzes Bündel von Maßnahmen vor, die auf eine strukturelle Transformation der kolumbianischen Gesellschaft hinarbeiten. Dies wird im Feld der Transitional Justice Studies seit geraumer Zeit unter dem Begriff der transformativen Gerechtigkeit (transformative justice) diskutiert. Das Spektrum der Maßnahmen, die auf eine Überwindung von Konfliktursachen zielen, ist - wie oben beschrieben - das Ergebnis eines jahrzehntelangen Kampfes von sozialen Bewegungen und zugleich Ausdruck eines transnationalen Normbildungsprozesses. Zugleich stellt sich demokratietheoretisch die brisante Frage, warum in einer stabilen elektoralen Demokratie (Diamond 1999) wesentliche Aspekte der sozial- und wohlfahrtsstaatlichen Ordnung des Landes zwischen der Regierung und 
einer bewaffneten Gruppe ausgehandelt werden sollten. Obwohl Landreformen, Drogenpolitik und Möglichkeiten der politischen Partizipation - unter Berücksichtigung einer Gender-, Differenz- und regionalen Perspektive - für die langfristige Transformation der sozialen, politischen und ökonomischen Strukturen des Landes und die Überwindung von Konfliktursachen fundamental sind, scheint das Vertragswerk als Ergebnis eines langjährigen Verhandlungsprozesses zu überambitioniert und dabei auch zu unbestimmt zu sein. Dies wird durch die Diskussion über den Stand der Implementierung bestätigt.

\section{Die erste Phase der Implementierung}

Fast dreieinhalb Jahre nach der Unterzeichnung des Friedensabkommens wäre es zwar vermessen, bereits eine abschließende Bilanz zu ziehen, gleichwohl lassen sich zur Umsetzung des Friedensabkommens aber erste Aussagen machen. Wie verschiedentlich nachgewiesen wurde, flammen bewaffnete Konflikte mit einer Wahrscheinlichkeit von 40-50\% innerhalb der ersten fünf Jahre nach dem Friedensschluss erneut auf und bewaffnete Konflikte, die auf dem Verhandlungsweg gelöst wurden, brechen dreimal so oft wieder aus wie militärisch beendete Konflikte (Westendorf 2015, S. 8). Die Diskussion über die Gründe ist facettenreich und reicht von der Frage der Architektur der Friedensabkommen und der Rolle externer Akteure bis hin zu Opportunitätskosten, Akkumulationsstrategien und Handlungsressourcen bewaffneter Akteure. In Kolumbien war die erste Phase der Implementierung des Abkommens mit der FARC-EP von Fehlstarts und Blockaden genauso geprägt wie von sichtbaren Erfolgen (McFee und Rettberg 2019). Über den Stand der Umsetzung des Abkommens berichten sowohl die Verifizierungsmission der Vereinten Nationen als auch das mit der Beobachtung der Umsetzung beauftragte Kroc Institute der University of Notre Dame regelmäßig ${ }^{14}$.

Im Prozess der Demobilisierung hatte zunächst die FARC-EP fast 9000 Waffen übergeben und inzwischen sind 13.104 Ex-Kämpfer*innen staatlich registriert, davon 2975 Frauen (Naciones Unidas 2020, S. 13, siehe auch den Beitrag von Barrios in diesem Heft). Die überwiegende Mehrheit von 9412 Ex-Kämpfer*innen lebt nicht (oder nicht mehr) in den ehemaligen Übergangszonen ETCR (Espacios Temporales de Capacitación y Reincorporación) und $27 \%$ haben Unterstützung für individuelle oder kollektive ökonomische Projekte erhalten (Naciones Unidas 2020, S. 6). Die FARC-EP wurde im August 2017 zur politischen Partei FARC (Fuerza Alternativa Revolucionaria del Común, dt. Alternative Revolutionäre Kraft des Volkes) und nahm unter erschwerten Bedingungen und mit bescheidenem Erfolg an den Wahlen des Jahres 2017 teil. Über den Möglichkeiten der politischen Partizipation und der zivilen Lebensweise hängt jedoch das Damoklesschwert der Gewalt. Zwischen der Unterzeichnung des Friedensschlusses und März 2020 wurden 190 Morde an ExKämpfer*innen registriert (darunter drei Frauen), 39 Mordversuche (darunter drei Frauen) und 13 Fälle von Verschwindenlassen (Naciones Unidas 2020, S. 9). Be-

\footnotetext{
14 Die Berichte der UN-Verifizierungsmission sind unter https://colombia.unmissions.org/documentos und
} die Berichte des Kroc Institute unter https://peaceaccords.nd.edu/barometer abrufbar (Stand: 10.05.2020). 
troffen sind vorwiegend ländliche Kontexte, die sich durch verbreitete Armut, kriminelle Ökonomien und die Präsenz bewaffneter Akteure auszeichnen. Auch wurden bis Ende 2019 landesweit 108 Morde an líderes y líderesas sociales (Aktivist*innen sozialer Bewegungen) registriert, darunter 15 Frauen und 2 LGBT Personen. Zur Entwicklung gender-basierter Gewalt lassen sich aufgrund der hohen Dunkelziffern nur Vermutungen anstellen. Die Zahl der registrierten Fälle ist hoch und schwankt regional (FIP 2019b). Die Gewaltbetroffenheit demobilisierter FARC-Kämpferinnen wird zwar als hoch eingeschätzt, doch empirische Belege sind ungenau (FIP 2019a; Avocats sans Frontières Canada und Humanas Colombia 2018).

Das Kroc Institute hat 2019 einen Bericht zum Stand der Umsetzung der genderbezogenen Bestimmungen des Friedensvertrages vorgelegt. Das Hauptergebnis der Analyse ist die Feststellung einer deutlichen Kluft zwischen der generellen Umsetzung des Friedensvertrages und der Umsetzung der genderspezifischen Maßnahmen. (Tab. 2).

Die Autor*innen des Berichts weisen darauf hin, dass einerseits 28 der Maßnahmen, die 2019 abgeschlossen sein sollten, noch nicht begonnen wurden, andererseits jedoch viele der Maßnahmen mittel- bis langfristig angelegt seien. Dennoch ist die hier konstatierte Kluft bemerkenswert.

Das Bild wird noch schärfer, wenn auch berücksichtigt wird, wie sich die Umsetzung der genderbezogenen Maßnahmen auf die verschiedenen Kapitel des Friedensvertrages auffächert. (Tab. 3).

Vor dem Hintergrund der oben entwickelten qualitativen Systematik der genderbezogenen Maßnahmen des Friedensabkommens ist hier zunächst zu unterstreichen, dass im August 2019 vor allem die mit der Implementierung und Verifizierung des Friedensvertrages und der Transitional Justice-Architektur verbundenen genderbezogenen Maßnahmen vollständig umgesetzt waren. Das Kroc Institute hebt in diesem Zusammenhang insbesondere Arbeitsgruppen und Koordinationen der Wahrheitskommission (CEV) und der Sondergerichtsbarkeit für den Frieden (JEP) hervor. Für die Umsetzung einer gender-sensiblen Agenda im Bereich des Umgangs mit systematisch begangenen Menschenrechtsverletzungen und Gewalttaten sind die inhaltliche Ebene und die tiefgehende und differenzierte Auseinandersetzung mit Gewaltbetroffenheit entscheidend. Um drei Beispiele zu nennen: Seit ihrer Einsetzung zeichnet sich erstens die Institution zur Suche nach Verwundenen (UBPD) durch eine starke Sensibilität für die Bedürfnisse von LGBT-Personen aus, für die oftmals biologische Familienstrukturen als beziehungsstiftendes Moment nachrangig sind. Wer registriert das Verschwinden von Trans*personen? Wer sucht nach ihnen?

Tab. 2 Stand der Umsetzung der Maßnahmen des Friedensvertrages, August 2019

\begin{tabular}{|c|c|c|}
\hline & $\begin{array}{l}\text { Genderbezogene Maßnahmen } \\
(\%)\end{array}$ & $\begin{array}{l}\text { Maßnahmen insgesamt } \\
(\%)\end{array}$ \\
\hline Noch nicht begonnen & 42 & 27 \\
\hline Minimale Umsetzung & 40 & 35 \\
\hline Mittlere Umsetzung & 10 & 13 \\
\hline Vollständige Umsetzung & 8 & 25 \\
\hline
\end{tabular}

Quelle: Kroc Institute (2019, S. 19) (Übers. AO) 
Tab. 3 Stand der Umsetzung der genderbezogenen Maßnahmen des Friedensvertrages, August 2019

\begin{tabular}{lllllll}
\hline & $\begin{array}{l}\text { Landreform } \\
(\%)\end{array}$ & $\begin{array}{l}\text { Partizipation } \\
(\%)\end{array}$ & $\begin{array}{l}\text { Konfliktende } \\
(\%)\end{array}$ & $\begin{array}{l}\text { Drogen } \\
(\%)\end{array}$ & $\begin{array}{l}\text { Opfer } \\
(\%)\end{array}$ & $\begin{array}{l}\text { Implementierung } \\
(\%)\end{array}$ \\
\hline $\begin{array}{l}\text { Nicht begonnen } \\
\text { Minimale }\end{array}$ & 59 & 35 & 33 & 47 & 18 & 17 \\
$\begin{array}{l}\text { Umsetzung } \\
\begin{array}{l}\text { Mittlere Um- } \\
\text { setzung }\end{array}\end{array}$ & 36 & 48 & 50 & 37 & 27 & 17 \\
$\begin{array}{l}\text { Vollständige } \\
\text { Umsetzung }\end{array}$ & 3 & 13 & 8 & 16 & 18 & 17 \\
\hline
\end{tabular}

Quelle: Kroc Institute (2019, S. 22) (Übers. AO)

Wer trauert? Wer ist Adressat*in von Trauerritualen? Zweitens zeichnet sich die Arbeit der Wahrheitskommission durch eine prominente intersektionale Perspektive aus. Von hoher symbolischer Bedeutung war der Umstand, dass die erste Großveranstaltung der Wahrheitskommission, die der Anerkennung von Gewalterfahrungen diente, im Juni 2019 in Cartagena unter dem Titel „Mi Cuerpo dice la Verdad“ (Mein Körper sagt die Wahrheit) stattfand. Es ging um die öffentliche Anerkennung der Systematik von gender-basierter Gewalt. Für die LGBT-community hatte diese Veranstaltung eine besondere Konnotation, denn ihre Sichtbarkeit im Friedensprozess hatte in den frühen 2000er Jahren mit dem Slogan „Cuerpo - Primer territorio de paz“" (Körper - erstes Territorium des Friedens) eingesetzt (Oettler 2019). Als drittes Beispiel für die Tiefe des Umgangs mit gender-basierter Gewalt kann die Praxis der Übergangsjustiz (JEP) genannt werden, die nicht nur Berichte von Betroffenen entgegennimmt, sondern insgesamt der Thematik der sexualisierten Gewalt hohe Priorität beimisst. Im Gegensatz zu diesen Entwicklungen ist zu konstatieren, dass die Umsetzung überall dort, wo es um die Überwindung der Konfliktursachen geht, nur schleppend verläuft.

Zwar sind auch im Bereich der Überwindung der Konfliktursachen Fortschritte zu verzeichnen, aber insgesamt übersetzt sich das unübersichtliche Panorama an Einzelmaßnahmen mit einem überwiegend geringen Konkretisierungsgrad (s. oben) in ein höchst komplexes Bild miteinander verschlungener friedens-, sozial- und entwicklungspolitischer Prozesse. Viele der genderbezogenen Maßnahmen sind gleichsam im Nationalen Entwicklungsplan der Regierung Duque vom Mai 2019 aufgegangen. Um die Unübersichtlichkeit des Panoramas an einem Beispiel zu veranschaulichen: inzwischen wurden in Kolumbien 170 Gemeinden (municipios) als Adressatinnen der PDET (Planes de Desarollo con Enfoque Territorial, dt: Entwicklungsplan mit territorialem Fokus) identifiziert. Eingebunden sind hier 11.000 veredas (dt: Weiler), 305 Gemeinderäte und 452 indigene Territorien (resguardos indígeneas) (Naciones Unidas 2020). In diesen 170 municipios wurden bisher 32.809 PATR-Initiativen (Plan Acción para Transformación Rural, dt. Aktionsplan zur ländlichen Transformation) entwickelt, von denen 1169 einen Gender-Bezug aufweisen und weitere 3239 das Potenzial haben, zu mehr Geschlechtergerechtigkeit beizutragen (Kroc Institute 2019, S. 31). Auch hier gilt: das Gesamtbild ist diffus, klare Kriterien und Indikatoren fehlen und eine Vielzahl von lokalen, regionalen und nationalen Akteuren ist beteiligt. 


\section{Fazit}

Während Friedensoptimist*innen durch die lokalen Konfliktdynamiken der letzten Jahre bereits auf eine harte Probe gestellt wurden, sind die Aussichten seit Ausbruch der Corona-Pandemie ungewisser denn je. Dies gilt für die Friedens- und Entwicklungsagenda im Allgemeinen und die Transformation von Geschlechterverhältnissen im Besonderen. Was zu befürchten steht, ist eine De-Priorisierung von gender-bezogenen Themen und eine verstärkte Sanktionierung von gender-Nonkonformität (O’Rourke 2020).

Im Vorangegangenen wurde aufgezeigt, wie der jahrzehntelange Kampf von Frauen-, feministischen und LGBT-Organisationen auf lokalen, nationalen und transnationalen Ebenen sowohl in prozeduraler als auch in substanzieller Hinsicht zur Verankerung von Gender im kolumbianischen Friedensprozess geführt hat. Damit ist Kolumbien Vorreiter einer feministischen Friedenspolitik, die Gendergerechtigkeit und die Überwindung von genderbezogenen Konfliktursachen und -folgen als conditio sine qua non eines dauerhaften und stabilen Friedens erachtet.

Die kolumbianische Erfahrung zeigt zweierlei. Erstens verweisen die Debatten um eine angeblich im Friedensvertrag vorhandene „Gender-Ideologie“ auf die Grenzen der sozialen Akzeptanz feministischer Anliegen. Der Begriff „Gender“ stand im Vorfeld der Volksabstimmung über den Friedensvertrag - wie auch in anderen Ländern - als ein Begriff im Kreuzfeuer der Kritik, der eine ,nicht-natürliche, damit also post-essentialistische Fassung von Geschlecht (und Sexualität)“ (Hark und Villa 2015, S. 7) beschreibt. Dass es bei der Revision des Vertragstextes vor allem darum ging, den Begriff zu umgehen und durch ein „,natürliches“ Verständnis von Geschlecht zu ersetzen, ist ein Rückschritt, andererseits ist es bemerkenswert, dass der Katalog der genderbezogenen Maßnahmen nicht eingedampft wurde.

Dieser umfangreiche Katalog von genderbezogenen Maßnahmen wurde in den obigen Abschnitten qualitativ systematisiert, um den Konkretisierungsgrad der Maßnahmen mit den inhaltlichen Achsen der Konfliktursachen, Konfliktfolgen und Konfliktbeilegung in Beziehung zu setzen. Die Analyse hat gezeigt, dass eine Häufung von wenig konkreten (und schwer zu operationalisierenden) Maßnahmen dort vorzufinden ist, wo es um die mittel- und langfristige Überwindung von Konfliktursachen - insbesondere Agrarreformen und Drogenanbau - geht. Dabei ist zu unterstreichen, dass das Gesamtbündel von Einzelmaßnahmen auf Forderungskataloge reagiert, die von sozialen Bewegungen über einen langen Zeitraum hinweg entwickelt und immer wieder in die Verhandlungsprozesse eingespeist wurden. So spiegeln sich in den genderbezogenen Einzelmaßnahmen vielfältige lokale Realitäten und Handlungsbedarfe.

Was sich im kolumbianischen Fall sehr deutlich zeigt, ist die Schwierigkeit, die für Mitglieder von Friedensverhandlungsdelegationen darin besteht, zwischen der Skylla der Überfrachtung und der Charybdis einer Priorisierung jenseits von lokalen Bedürfnissen zu navigieren. Dies gilt - wie oben betont - für die Frage der Einbeziehung einer genderbezogenen Perspektive wie auch insgesamt für die Schaffung eines inklusiven Friedens. Wie Madhav und Quinn (2015) gezeigt haben, ziehen Friedensverträge, die mehrere Politikfelder abdecken, nicht zwangsläufig weniger 
bewaffnete Konflikte nach sich. Wichtiger als die Breite der Maßnahmen scheint die Einbeziehung von besonders wichtigen Maßnahmen zu sein (ebda.).

Der kolumbianische Fall zeigt sehr deutlich, dass einige Maßnahmen für die Fortentwicklung der Gender-Perspektive zentral sind. Dazu zählen die konkreten Arbeitsgruppen innerhalb des Transitional-Justice-Systems und die 2017 gegründete Instancia de Alto Nivel de Gobierno para la Incorporación del Enfoque de Género (dt.: Hochrangige Regierungsinstanz zur Einbeziehung des Gender-Fokus). Wie die Phase des pos-acuerdo, die turbulente Zeit nach dem Friedensvertrag, gezeigt hat, schafft die Vertragsunterzeichnung lediglich eine Ausgangsbedingung für die weitere Politikgestaltung. Die Einsicht, dass dauerhafter Frieden den Schutz vor genderbasierter Gewalt und einen Wandel der Gewaltkultur voraussetzt, ist damit indes noch nicht fest verankert.

Funding Open Access funding enabled and organized by Projekt DEAL.

Open Access Dieser Artikel wird unter der Creative Commons Namensnennung 4.0 International Lizenz veröffentlicht, welche die Nutzung, Vervielfältigung, Bearbeitung, Verbreitung und Wiedergabe in jeglichem Medium und Format erlaubt, sofern Sie den/die ursprünglichen Autor(en) und die Quelle ordnungsgemäß nennen, einen Link zur Creative Commons Lizenz beifügen und angeben, ob Änderungen vorgenommen wurden.

Die in diesem Artikel enthaltenen Bilder und sonstiges Drittmaterial unterliegen ebenfalls der genannten Creative Commons Lizenz, sofern sich aus der Abbildungslegende nichts anderes ergibt. Sofern das betreffende Material nicht unter der genannten Creative Commons Lizenz steht und die betreffende Handlung nicht nach gesetzlichen Vorschriften erlaubt ist, ist für die oben aufgeführten Weiterverwendungen des Materials die Einwilligung des jeweiligen Rechteinhabers einzuholen.

Weitere Details zur Lizenz entnehmen Sie bitte der Lizenzinformation auf http://creativecommons.org/ licenses/by/4.0/deed.de.

\section{Literatur}

Avocats sans Frontières Canada, und Humanas Colombia. 2018. Situación de las mujeres rurales y mujeres ex combatientes en el marco del Acuerdo Final de Paz. https:/www.humanas.org.co/ alfa/dat_particular/ar/arc_84559_q_Situacion_de_las_mujeres_rurales_y_excombatientes_en_el_ marco_del_Acuerdo_de_Paz.pdf. Zugegriffen: 21. Dezember 2020.

Beltrán, William Mauricio, und Siam Creely. 2018. Pentecostals, Gender Ideology and the Peace Plebiscite: Colombia 2016. Religions 8(12):1-19

Bouvier, Virginia M. 2016. Gender and the role of women in Colombia's peace process. New York: UN Women.

Castillo Vargas, Elizabeth. 2019. No somos etcétera. Veinte años de historia del movimiento LGBT en Colombia. Bogotá: Penguin Random House.

Centro Nacional de Memoria Histórica. 2018. Todo pasó frente a nuestros ojos. El genocidio de la Unión Patriótica 1984-2002, Bogotá: CNMH. https://www.jep.gov.co/Sala-de-Prensa/Documents/ INFORME_UP_completo.pdf. Zugegriffen: 21. Dezember 2020.

Céspedes-Baéz, Lina M. 2017. En los confines de lo posible: inclusión del enfoque de género en el Acuerdo de La Habana. In Utopía u oportunidad fallida. Análisis Crítico del Acuerdo de Paz, Hrsg. Lina M. Céspedes-Baéz, Enrique Prieto Rios, 295-326. Bogotá: Editorial Universidad del Rosario.

Céspedes-Baéz, Lina M., und Felipe Jaramillo Ruiz. 2018. 'Peace without women does not go!' Women's struggle for inclusion in Colombia's peace process with the FARC. Colombia Internacional 94:83-109

Chaparro González, Nina, und Margarita Martínez Osorio. 2016. Negociando desde los márgenes: la participación política de las mujeres en los procesos de paz en Colombia (1982-2016). Bogotá: Dejusticia. 
Chernick, Mark. 1999. Negotiating peace amid multiple forms of violence: the protracted search for a settlement to the armed conflicts in Colombia. In Comparative peace processes in Latin America, Hrsg. Cynthia Arnson, 159-195. Washington: Woodrow Wilson Center Press.

Connell, R. 1987. Gender and power. Sydney: Allen \& Unwin.

Corporación Humanas, und Corporación de Investigación y Acción Social y Económica. 2017. Viviencias, Aportes y Reconocimiento: Las Mujeres en el Proceso de Paz en la Habana. Bogotá: Corporación Humanas/ CIASE.

Cumbre Nacional de Mujeres por la Paz. 2014. Sistematización del Cumbre, Bogotá 23-25 Octobre 2013. https://www2.unwomen.org/-/media/field\%20office\%20colombia/documentos/publicaciones/2014/ sistematizacumbre_mujeres_y_paz.pdf?la=es\&vs=2850. Zugegriffen: 21. Dezember 2020.

Diamond, Larry. 1999. Developing democracy. Toward consolidation. Baltimore: Johns Hopkins University Press.

Esguerra Muelle, Camila. 2017. Cómo hacer necropolíticas en casa: Ideología de género y acuerdos de paz en Colombia. Sexualidad, Salud y Sociedad 27:172-189.

FIP - Fundación Ideas para la Paz. 2019a. Las trayectorias de la reincorporación y la seguridad de los excombatientes de las FARC. Riesgos, respuestas del Estado y tareas pendientes. http://ideaspaz.org/ media/website/FIP_NE_TrayectoriasFarc_Final_V02.pdf. Zugegriffen: 21. Dezember 2020.

FIP - Fundación Ideas para la Paz. 2019b. Las violencias de género en la transición legado del conflicto armado y desaíos para la paz. http://www.ideaspaz.org/media/website/FIP_SerieLGBTI_Docu_ estrategico.pdf. Zugegriffen: 21. Dezember 2020.

Gobierno Nacional, und FARC-EP. 2016a. Acuerdo Final para la Terminación del Conflicto y la Construcción de una Paz Estable y Duradera (AI). 24. August 2016. https://draftable.com/compare/ JjypTOknafBktqvc. Auch http://www.indepaz.org.co/acuerdo-final-para-la-terminacion-del-conflic toy-la-construccion-de-una-paz-estable-y-duradera/. Zugegriffen: 21. Dezember 2020.

Gobierno Nacional, und FARC-EP. 2016b. Acuerdo Final para la Terminación del Conflicto y la Construcción de una Paz Estable y Duradera (AII). 12. November 2016. https://draftable.com/compare/ JjypTOknafBktqvc. Auch https://www.unidadvictimas.gov.co/es/acuerdo-final-para-la-terminaciondel-conflicto-y-la-construccion-de-una-paz-estable-y-duradera. Zugegriffen: 21. Dezember 2020.

Hagen, Jamie J. 2016. Queering women, peace and security. International Affairs 92(2):313-332.

Hark, Sabine, und Paula-Irene Villa (Hrsg.). 2015. Anti-Genderismus: Sexualität und Geschlecht als Schauplätze aktueller politischer Auseinandersetzungen. Bielefeld: transcript.

Jäger, Siegfried. 2004. Kritische Diskursanalyse. Eine Einführung. 4. Aufl. Münster: Unrast.

Jaramillo Paz, Leslie, und Germán Darío Valencia Agudelo. 2015. Atipicidades del proceso de paz con las Milicias Populares de Medellín. Estudios Políticos 46:263-282.

Joshi, Madhav, und Michael J. Quinn. 2015. Is the sum greater than the parts? The terms of civil war peace agreements and the commitment problem revisited. Negotiation Journal 31(1):7-30.

Kroc Institute. 2019. Gender equality for sustainable peace. Second report on the monitoring of the gender perspective in the implementation of the Colombian peace accord. http://peaceaccords.nd.edu/ wp-content/uploads/2020/04/012820-GENDER-REPORT-DIGITAL.pdf. Zugegriffen: 21. Dezember 2020 .

Kurtenbach, Sabine. 2001. Kann Kolumbien aus seiner eigenen Geschichte lernen? In Kolumbien zwischen Gewalteskalation und Friedenssuche. Möglichkeiten und Grenzen der Einflussnahme externer Akteure, Hrsg. Sabine Kurtenbach, 93-109. Frankfurt a.M.: Vervuert.

Landwehr, Achim. 2008. Historische Diskursanalyse. Frankfurt/ New York: Campus.

Lee, Sung Yong, Roger Mac Ginty, und Madhav Joshi. 2016. Social peace versus security peace. Global Governance 22(4):491-512.

McFee, Erin, und Angelika Rettberg. 2019. Contexto de los deafíos de la implementación temprana en Colombia. In Excombatientes y acuerdo de paz con las FARC-EP en Colombia: balance de la etapa temprana, Hrsg. Erin McFee, Angelika Rettberg, 1-18. Bogotá: Ediciones Uniandes.

Naciones Unidas. 2020. Misión de Verificación de las Naciones Unidas en Colombia. Informe del Secretario General S/2020/239 (26.03.2020). https://colombia.unmissions.org/sites/default/files/sp_ n2007155_0.pdf. Zugegriffen: 21. Dezember 2020.

Oettler, Anika. 2004. Erinnerungsarbeit und Vergangenheitspolitik in Guatemala. Frankfurt a. M.: Vervuert.

Oettler, Anika. 2016. Gender Trouble im Friedensprozess. an.schläge 8: 10-11.

Oettler, Anika. 2019. The Struggle for Gendered Peace and LGBT Rights in Colombia, London School of Economics: LSE-GIGA-CCDP Violence, Security, and Peace Working Papers No.2. https://www. 1se.ac.uk/lacc/publications/PDFs/VSP2-Oettler-Gendered-Peace-LGBT-Rights-Colombia-web.pdf. Zugegriffen 21. Dezember 2020. 
ONU Mujeres. 2018. 100 medidas que incorporan la perspectiva de género en el Acuerdo de Paz entre el Gobierno de Colombia y las FARC-EP para terminar el conflicto y construir una paz estable y duradera. https://colombia.unwomen.org/es/biblioteca/publicaciones/2017/05/100-medidas-generoacuerdos. Zugegriffen: 21. Dezember 2020.

O'Rourke, Catherine. 2020. COVID-19 and gender-based violence in conflict: new challenges and persistent problems. Political settlements research programme, 20 April 2020. http://www.politicalsettle ments.org/2020/04/21/covid-19-and-gender-based-violence-in-conflict/. Zugegriffen: 21. Dezember 2020.

Presidencia de la República, und Oficina del Alto Comisionado para la Paz. 2018. Biblioteca del Proceso de Paz con las FARC-EP. Tomo VII. http://www.altocomisionadoparalapaz.gov.co/Documents/ biblioteca-proceso-paz-farc/tomo-7-proceso-paz-farc-mecanismos-participacion-mesa-conversacio nes.pdf. Zugegriffen: 21. Dezember 2020.

Quintero, Beatriz. 2003. Cartografía de las mujeres. Bogotá: Red Nacional de Mujeres.

Rojas, Catalina. 2009. Women and peacebuilding in Colombia: resistance to war, creativity for peace. In Colombia: building peace in a time of war, Hrsg. Virginia M. Bouvier, 207-224. Washington: United States Institute of Peace Press.

Ruppert, Uta. 1998. Geschlechterverhältnisse in der Internationalen Politik. Eine Einführung. In Lokal bewegen, global handeln. Internationale Politik und Geschlecht, Hrsg. Uta Ruppert, 7-23. Frankfurt a.M.: Campus.

Salvesen, Hilde, und Dag Nylander. 2017. Towards an inclusive peace: women and the gender approach in the Colombian peace process. https://noref.no/Publications/Regions/Colombia/Towardsan-inclusive-peace-women-and-the-gender-approach-in-the-Colombian-peace-process. Zugegriffen: 21.Dezember 2020.

Tate, Winifred. 2009. From greed to grievance: the shifting political profile of the Colombian paramilitaries. In Colombia: building peace in a time of war, Hrsg. Virginia M. Bouvier, 111-131. Washington: United States Institute of Peace Press.

Theidon, Kimberly. 2009. Reconstructing masculinities: the disarmament, demobilization, and reintegration of former combatants in Colombia. Human Rights Quarterly 31(1):1-34.

UN Sicherheitsrat. 2000. Resolution 1325 (31.10.2000). https://www.un.org/depts/german/sr/sr_00/ sr1325.pdf. Zugegriffen: 21. Dezember 2020.

Villarraga Sarmiento, Álvaro (Hrsg.). 2013. Movimiento Ciudadano y Social por la Paz. Biblioteca de la Paz 1980-2013, Bd. IX. Bogotá: Fundación Cultura Democrática.

Wasmuth, Ulrike C. 1998. Die Erfindung der Kategorie Geschlecht oder: wie männlich ist die Friedensforschung? In Lokal bewegen, global handeln. Internationale Politik und Geschlecht, Hrsg. Uta Ruppert, 56-76. Frankfurt a.M: Campus.

Westendorf, Jasmine-Kim. 2015. Why peace processes fail: negotiating insecurity after civil war. Boulder: Lynne Rienner.

Zulaga Nieto, Jaime. 2013. El movimiento por la paz y contra la guerra. In Movimiento Ciudadano y Social por la Paz. Biblioteca de la Paz 1980-2013, Bd. IX, Hrsg. Álvaro Villarraga Sarmiento, 41-55. Bogotá: Fundación Cultura Democrática. 